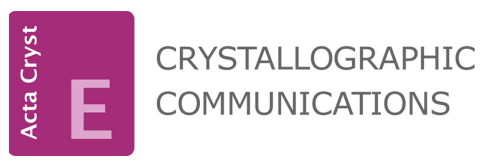

ISSN 2056-9890

Received 13 September 2015

Accepted 6 October 2015

Edited by $\mathrm{H}$. Stoeckli-Evans, University of Neuchâtel, Switzerland

Keywords: crystal structure; triazine; cyclohexanol; channel inclusion; Piedfort units; hydrogen bonding

CCDC reference: 1430153

Supporting information: this article has supporting information at journals.iucr.org/e

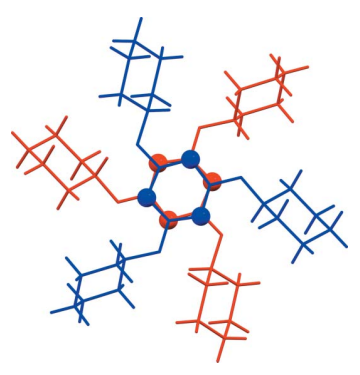

OPEN $\odot$ ACCESS

\section{Crystal structure of 2,4,6-tris(cyclohexyloxy)-1,3,5- triazine}

\author{
Ravish Sankolli, ${ }^{\text {jürg Hauser, }}{ }^{\text {a }}$ T. N. Guru Row ${ }^{\mathrm{b}}$ and Jürg Hulliger ${ }^{\mathrm{a} *}$ \\ ${ }^{a}$ Department of Chemistry and Biochemistry, University of Bern, Freiestrasse 3, CH-3012 Bern, Switzerland, and ${ }^{\mathbf{b}}$ Solid \\ State and Structural Chemistry Unit, Indian Institute of Science, Bangalore 560 012, Karnataka, India. *Correspondence \\ e-mail: juerg.hulliger@dcb.unibe.ch
}

The title compound, $\mathrm{C}_{21} \mathrm{H}_{33} \mathrm{~N}_{3} \mathrm{O}_{3}$, is a tri-substituted cyclohexyloxy triazine. In the crystal, the triazine rings form (C3i-PU) Piedfort units. The inter-centroid distance of the $\pi-\pi$ interaction involving the triazine rings is 3.3914 (10) $\AA$. In the crystal, molecules are linked by $\mathrm{C}-\mathrm{H} \cdots \mathrm{O}$ hydrogen bonds, forming ribbons propagating along $[1 \overline{1} 0]$. There are also weak $\mathrm{C}-\mathrm{H} \cdots \mathrm{N}$ and $\mathrm{C}-\mathrm{H} \cdots \mathrm{O}$ contacts present, linking inversion-related ribbons, forming a three-dimensional structure.

\section{Chemical content}

Cyclohexyl derivatives are known to have applications in various fields of chemistry. The mono- and di-substituted derivatives of triazine with cyclohexanol show antiviral activity (Mibu et al., 2013), wherein cyclohexyl esters show the properties of traction fluids (Baldwin et al., 1997). Partially substituted menthoxy triazines can be used as enantio-differentiating reagents in organic synthesis (Kamiński et al., 1998). The cyclohexyl trimer, perhydrotriphenelene (PHTP) can form inclusion compounds showing non-linear optical properties (Hoss et al., 1996). In particular, PHTP as a renowned host in the literature, forms variable inclusions with functional molecules (Allegra et al., 1967; König et al., 1997; Couderc \& Hulliger, 2010). Most triazines also exhibit various types of inclusion properties (Süss et al., 2002, 2005; Reichenbächer $e t$ al., 2004). Thus, the title compound was synthesized to study the supramolecular features in comparison to PHTP. Symmetrically substituted triazines with three cyclohexanol units through an oxygen linkage shows a trigonal symmetry in its trans racemic form and a planar geometry in its crystal structure. So far, the crystallization of the title compound with conventional solvents did not form any inclusions. To the best of our knowledge, this is the first tri-substituted cyclohexyloxy triazine to be described.






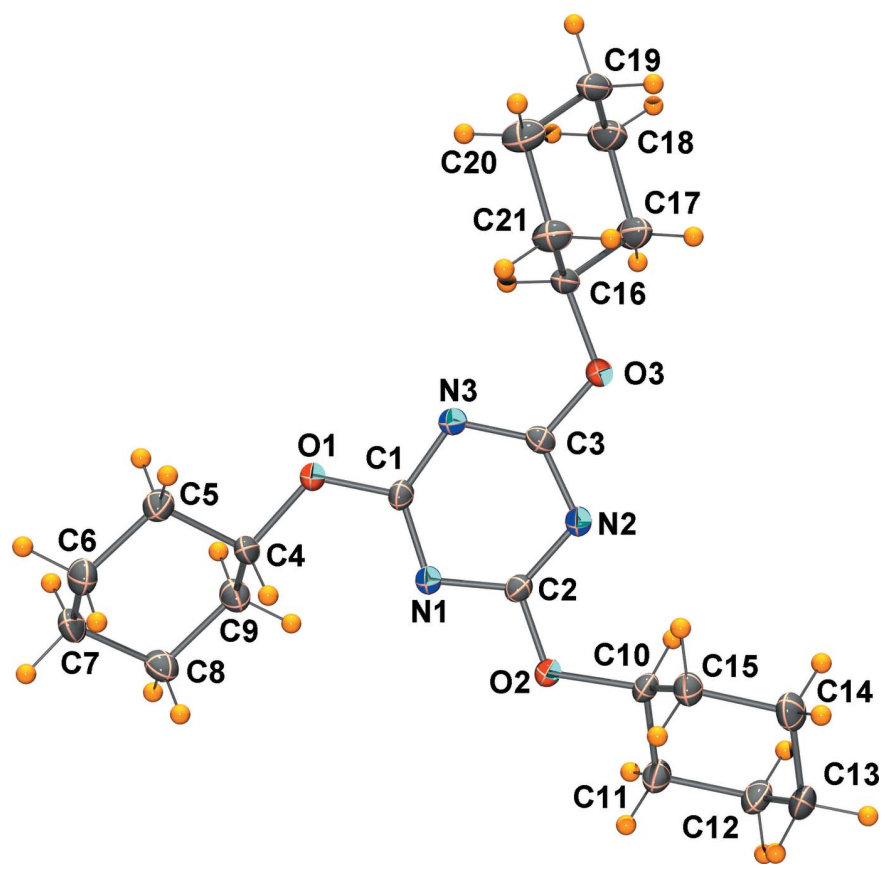

Figure 1

The molecular structure of the title compound, with atom labelling. Displacement ellipsoids drawn at the $50 \%$ probability level. The $\mathrm{C}-\mathrm{O}-$ $\mathrm{C}=\mathrm{N}$ torsion angles are $\mathrm{C} 4-\mathrm{O} 1-\mathrm{C} 1-\mathrm{N} 1=3.6(2), \mathrm{C} 10-\mathrm{O} 2-\mathrm{C} 2-\mathrm{N} 2$ $=-1.2(2)$ and $\mathrm{C} 16-\mathrm{O} 3-\mathrm{C} 3-\mathrm{N} 3=-3.1(2)^{\circ}$.

\section{Structural commentary}

The molecular structure of the title compound is illustrated in Fig. 1. The molecule has threefold rotation symmetry, but there are small variation in the $\mathrm{C}-\mathrm{O}-\mathrm{C}=\mathrm{N}$ torsion angles; $\mathrm{C} 4-\mathrm{O} 1-\mathrm{C} 1-\mathrm{N} 1=3.6(2), \mathrm{C} 10-\mathrm{O} 2-\mathrm{C} 2-\mathrm{N} 2=-1.2(2)$ and $\mathrm{C} 16-\mathrm{O} 3-\mathrm{C} 3-\mathrm{N} 3=-3.1(2)^{\circ}$.

\section{Supramolecular features}

In the crystal, molecules are linked by $\mathrm{C}-\mathrm{H} \cdots \mathrm{O}$ hydrogen



Table 1

Hydrogen-bond geometry $\left(\AA,^{\circ}\right)$.

\begin{tabular}{lllll}
\hline$D-\mathrm{H} \cdots A$ & $D-\mathrm{H}$ & $\mathrm{H} \cdots A$ & $D \cdots A$ & $D-\mathrm{H} \cdots A$ \\
\hline $\mathrm{C} 12-\mathrm{H} 12 A \cdots \mathrm{O} 1^{\mathrm{i}}$ & 0.99 & 2.45 & $3.413(2)$ & 164 \\
$\mathrm{C} 9-\mathrm{H} 9 A \cdots \mathrm{O}^{\mathrm{ii}}$ & 0.99 & 2.60 & $3.528(2)$ & 156 \\
$\mathrm{C} 10-\mathrm{H} 10 \cdots \mathrm{O} 1^{\mathrm{ii}}$ & 1.00 & 2.95 & $3.787(2)$ & 142 \\
$\mathrm{C} 5-\mathrm{H} 5 B \cdots \mathrm{N} 1^{\mathrm{iii}}$ & 0.99 & 2.77 & $3.684(2)$ & 154 \\
\hline Symmetry codes: $\quad$ (i) & $x-1, y-1, z ; \quad$ (ii) & $-x+2,-y+2,-z+1 ;$ & (iii) \\
$-x+3,-y+2,-z+1$. & & & &
\end{tabular}

Table 1). Inversion-related ribbons are linked by weak $\mathrm{C}-$ $\mathrm{H} \cdots \mathrm{N}$ and $\mathrm{C}-\mathrm{H} \cdots \mathrm{O}$ contacts, forming a three-dimensional structure (Table 1). There are Piedfort units (C3i-PU) present (Jessiman et al., 1990), as shown in Fig. 3. The inter-centroid distance of the slightly slipped parallel $\pi-\pi$ interaction involving inversion-related triazine rings is 3.3914 (10) $\AA$. The inter-planar distance is 3.3315 (7) $\AA$, while the slippage is $0.634 \AA$. There are three $\mathrm{C}-\mathrm{H} \cdots \mathrm{H}-\mathrm{C}$ van der Waals contacts, $2.28,2.28$ and $2.37 \AA$, which are longer than those in the crystal structure of PHTP (measured 2.13, 2.14 and $2.16 \AA$; Harlow \& Desiraju, 1990).

The perhydrogenated outer wall resembles the structural features of PHTP (pehydrotriphenylene) in its crystal structure with $\mathrm{C}-\mathrm{H} \cdots \mathrm{H}-\mathrm{C}$ short contacts (Harlow \& Desiraju, 1990). In comparison, PHTP is a highly symmetrical chiral molecule, which is used for inclusions in its all-trans racemic form (König et al., 1997). Thus, the title compound is a perhydrogenated triazine analogue of PHTP. However, the triazine rings which form Piedfort units (Jessiman et al., 1990) and the $\mathrm{C}-\mathrm{H} \cdots \mathrm{O}$ and $\mathrm{C}-\mathrm{H} \cdots \mathrm{N}$ hydrogen bonds (Table 1) contribute to the stabilization of the structure as compared to PHTP.

\section{Synthesis and crystallization}

Cyclohexanol $(10.4 \mathrm{ml}, 10.02 \mathrm{~g}, 100 \mathrm{mmol})$ and sodium hydride $(2.88 \mathrm{~g}, 120 \mathrm{mmol})$ were taken in a round bottom flask

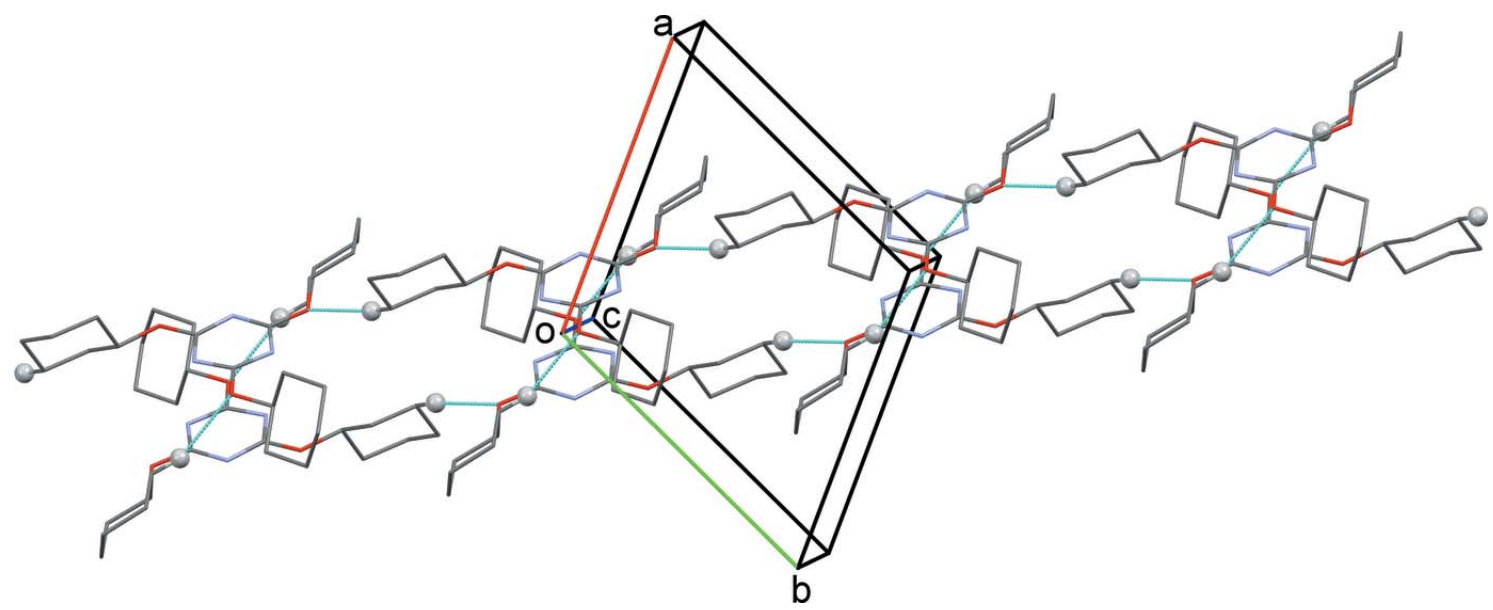

Figure 2

A view along the $c$ axis of the crystal packing of the title compound. The most significant $\mathrm{C}-\mathrm{H} \cdots \mathrm{O}$ hydrogen bonds (see Table 1 ) are shown as dashed lines, and the only $\mathrm{H}$ atoms shown are $\mathrm{H} 12 A$ and $\mathrm{H} 9 A$ (grey balls) for clarity. 


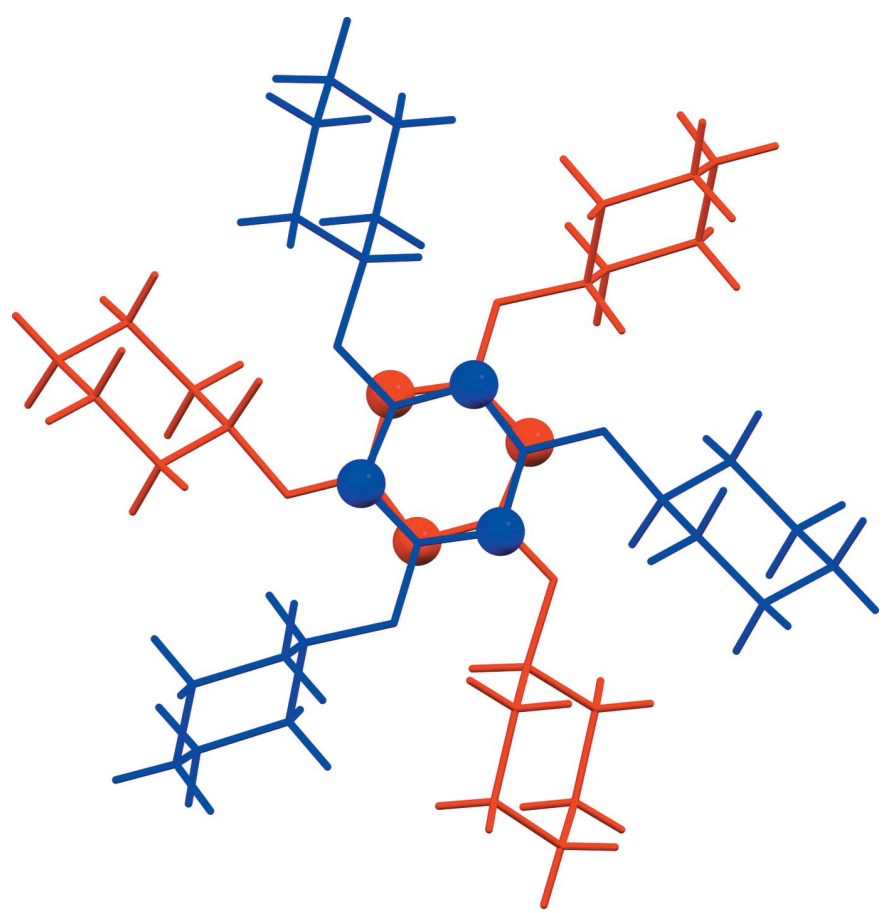

Figure 3

A view of the Piedfort unit (C3i-PU), with the two triazine rings stacking one above the other, forming an hexagonal symmetry unit. The $\mathrm{N}$ atoms are shown as red and blue balls.

containing $50 \mathrm{ml}$ of THF at $273 \mathrm{~K}$. The mixture was stirred at room temperature for $30 \mathrm{~min}$, then cyanuric chloride $(4.6 \mathrm{~g}$, $25 \mathrm{mmol}$ ) was carefully added in one portion. The mixture was stirred overnight at $323 \mathrm{~K}$. The solvent was then removed under reduced pressure and the oily mixture was transferred in to a separating funnel and extracted with $\mathrm{CH}_{2} \mathrm{Cl}_{2}(3 \times$ $100 \mathrm{ml}$ ). Again, the solvent was removed under reduced pressure and the crude product was further purified through column chromatography $\left(\mathrm{SiO}_{2}\right.$ 60, eluent: diethyl ether/ pentane 1:1) to yield the pure product as a white powder. Colourless prismatic crystals were obtained by isothermal evaporation of a solution in THF.

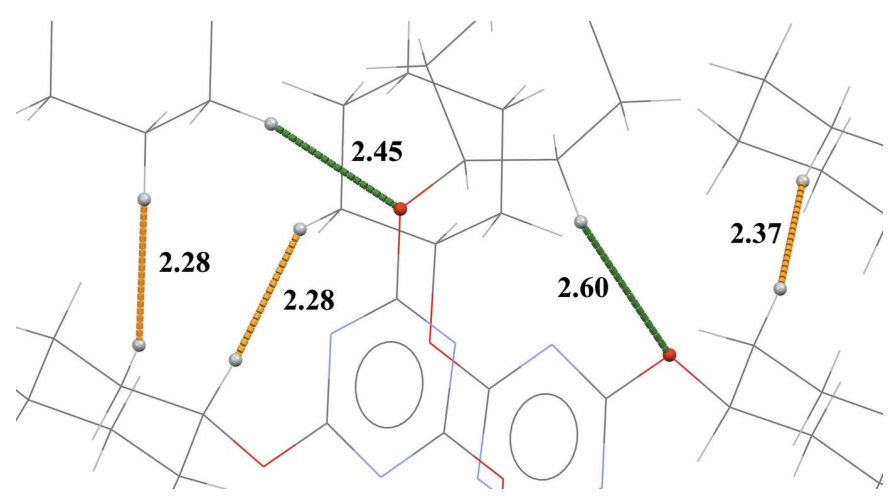

Figure 4

A view of the short $\mathrm{C}-\mathrm{H} \cdots \mathrm{H} \cdots \mathrm{C}$ contacts (orange dashed lines) and some $\mathrm{C}-\mathrm{H} \cdots \mathrm{O}$ hydrogen bonds (green dashed lines; see Table 1 ) in the crystal structure of the title compound.
Table 2

Experimental details.

\begin{tabular}{|c|c|}
\hline \multicolumn{2}{|l|}{ Crystal data } \\
\hline Chemical formula & $\mathrm{C}_{21} \mathrm{H}_{33} \mathrm{~N}_{3} \mathrm{O}_{3}$ \\
\hline$M_{\mathrm{r}}$ & 375.50 \\
\hline Crystal system, space group & Triclinic, $P \overline{1}$ \\
\hline Temperature $(\mathrm{K})$ & 100 \\
\hline$a, b, c(\AA)$ & $9.7020(2), 10.1456(3), 11.2064(3)$ \\
\hline$\alpha, \beta, \gamma\left({ }^{\circ}\right)$ & $96.528(2), 95.982(2), 112.110(2)$ \\
\hline$V\left(\AA^{3}\right)$ & $1002.30(5)$ \\
\hline$Z$ & 2 \\
\hline Radiation type & Mo $K \alpha$ \\
\hline$\mu\left(\mathrm{mm}^{-1}\right)$ & 0.08 \\
\hline Crystal size $(\mathrm{mm})$ & $0.47 \times 0.24 \times 0.10$ \\
\hline \multicolumn{2}{|l|}{ Data collection } \\
\hline Diffractometer & Agilent SuperNova, Eos \\
\hline Absorption correction & $\begin{array}{l}\text { Multi-scan (CrysAlis PRO; } \\
\text { Agilent, 2014) }\end{array}$ \\
\hline$T_{\min }, T_{\max }$ & $0.657,1$ \\
\hline $\begin{array}{l}\text { No. of measured, independent and } \\
\text { observed }[I>2 \sigma(I)] \text { reflections }\end{array}$ & $24791,4106,3603$ \\
\hline$R_{\text {int }}$ & 0.027 \\
\hline$(\sin \theta / \lambda)_{\max }\left(\AA^{-1}\right)$ & 0.625 \\
\hline \multicolumn{2}{|l|}{ Refinement } \\
\hline$R\left[F^{2}>2 \sigma\left(F^{2}\right)\right], w R\left(F^{2}\right), S$ & $0.052,0.142,1.04$ \\
\hline No. of reflections & 4106 \\
\hline No. of parameters & 244 \\
\hline $\mathrm{H}$-atom treatment & $\mathrm{H}$-atom parameters constrained \\
\hline$\Delta \rho_{\max }, \Delta \rho_{\min }\left(\mathrm{e} \AA^{-3}\right)$ & $0.61,-0.21$ \\
\hline
\end{tabular}

Computer programs: CrysAlis PRO (Agilent, 2014), SHELXS2014/7 (Sheldrick, 2008), SHELXL2014/7 (Sheldrick, 2015), POV-RAY (POV-RAY Team, 2004), Mercury (Macrae et al., 2008), and PLATON (Spek, 2009).

\section{Refinement}

Crystal data, data collection and structure refinement details are summarized in Table 2. The $\mathrm{C}$-bound $\mathrm{H}$ atoms were included in calculated positions and treated as riding atoms: $\mathrm{C}-\mathrm{H}=0.99-1.00 \AA$ with $U_{\text {iso }}(\mathrm{H})=1.2 U_{\text {eq }}(\mathrm{C})$.

\section{Acknowledgements}

This work was supported by the Swiss National Science Foundation through the Indo-Swiss Joint Research Program (project number ISJRP 138 860).

\section{References}

Agilent (2014). CrysAlis PRO. Agilent Technologies, Yarnton, UK. Allegra, G., Farina, M., Immirzi, A., Colombo, A., Rossi, U., Broggi, I. \& Natta, G. (1967). J. Chem. Soc. B, pp. 1020-1028.

Baldwin, C. R., Britton, M. M., Davies, S. C., Gillies, D. G., Hughes, D. L., Smith, G. W. \& Sutcliffe, L. H. (1997). J. Mol. Struct. 403, 116.

Couderc, G. \& Hulliger, J. (2010). Chem. Soc. Rev. 39, 1545-1554.

Harlow, R. L. \& Desiraju, G. R. (1990). Acta Cryst. C46, 1054-1055.

Hoss, R., König, O., Kramer-Hoss, V., Berger, P., Rogin, P. \& Hulliger, J. (1996). Angew. Chem. Int. Ed. Engl. 35, 1664-1666.

Jessiman, A. S., MacNicol, D. D., Mallinson, P. R. \& Vallance, I. (1990). J. Chem. Soc. Chem. Commun. pp. 1619-1621.

Kamiński, Z. J., Markowicz, S. W., Kolesińska, B., Martynowski, D. \& Główka, M. L. (1998). Synth. Commun. 28, 2689-2696.

König, O., Bürgi, H. B., Armbruster, T., Hulliger, J. \& Weber, T. (1997). J. Am. Chem. Soc. 119, 10632-10640. 
Macrae, C. F., Bruno, I. J., Chisholm, J. A., Edgington, P. R., McCabe, P., Pidcock, E., Rodriguez-Monge, L., Taylor, R., van de Streek, J. \& Wood, P. A. (2008). J. Appl. Cryst. 41, 466-470.

Mibu, N., Yokomizo, K., Takemura, S., Ueki, N., Itohara, S., Zhou, J., Miyata, T. \& Sumoto, K. (2013). Chem. Pharm. Bull. 61, 823833.

POV-RAY Team (2004). POV-RAY - The Persistance of Vision Raytracer. http://www.povray.org
Reichenbächer, K., Süss, H. I., Stoeckli-Evans, H., Bracco, S., Sozzani, P., Weber, E. \& Hulliger, J. (2004). New J. Chem. 28, 393-397. Sheldrick, G. M. (2008). Acta Cryst. A64, 112-122.

Sheldrick, G. M. (2015). Acta Cryst. C71, 3-8.

Spek, A. L. (2009). Acta Cryst. D65, 148-155.

Süss, H. I., Lutz, M. \& Hulliger, J. (2002). CrystEngComm, 4, 610-612. Süss, H. I., Neels, A. \& Hulliger, J. (2005). CrystEngComm, 7, 370373. 


\section{supporting information}

Acta Cryst. (2015). E71, 1328-1331 [https://doi.org/10.1107/S2056989015018782]

\section{Crystal structure of 2,4,6-tris(cyclohexyloxy)-1,3,5-triazine}

\section{Ravish Sankolli, Jürg Hauser, T. N. Guru Row and Jürg Hulliger}

Computing details

Data collection: CrysAlis PRO (Agilent, 2014); cell refinement: CrysAlis PRO (Agilent, 2014); data reduction: CrysAlis PRO (Agilent, 2014); program(s) used to solve structure: SHELXS2014/7 (Sheldrick, 2008); program(s) used to refine structure: SHELXL2014/7 (Sheldrick, 2015); molecular graphics: POV-RAY (POV-RAY Team, 2004) and Mercury (Macrae et al., 2008); software used to prepare material for publication: SHELXL2014/7 (Sheldrick, 2015) and PLATON (Spek, 2009).

\section{2,4,6-Tris(cyclohexyloxy)-1,3,5-triazine}

\section{Crystal data}

$\mathrm{C}_{21} \mathrm{H}_{33} \mathrm{~N}_{3} \mathrm{O}_{3}$

$M_{r}=375.50$

Triclinic, $P \overline{1}$

$a=9.7020(2) \AA$

$b=10.1456(3) \AA$

$c=11.2064(3) \AA$

$\alpha=96.528(2)^{\circ}$

$\beta=95.982(2)^{\circ}$

$\gamma=112.110(2)^{\circ}$

$V=1002.30(5) \AA^{3}$

\section{Data collection}

Agilent SuperNova, Eos diffractometer

Radiation source: Mo X-ray Source Detector resolution: 16.0965 pixels $\mathrm{mm}^{-1}$ $\omega$ scans

Absorption correction: multi-scan

(CrysAlis Pro; Agilent, 2014)

$T_{\min }=0.657, T_{\max }=1$

Refinement

Refinement on $F^{2}$

Least-squares matrix: full

$R\left[F^{2}>2 \sigma\left(F^{2}\right)\right]=0.052$

$w R\left(F^{2}\right)=0.142$

$S=1.04$

4106 reflections

244 parameters

0 restraints
$Z=2$

$F(000)=408$

$D_{\mathrm{x}}=1.244 \mathrm{Mg} \mathrm{m}^{-3}$

Mo $K \alpha$ radiation, $\lambda=0.71073 \AA$

Cell parameters from 9471 reflections

$\theta=2.2-27.7^{\circ}$

$\mu=0.08 \mathrm{~mm}^{-1}$

$T=100 \mathrm{~K}$

Prism, colourless

$0.47 \times 0.24 \times 0.10 \mathrm{~mm}$

24791 measured reflections

4106 independent reflections

3603 reflections with $I>2 \sigma(I)$

$R_{\text {int }}=0.027$

$\theta_{\max }=26.4^{\circ}, \theta_{\min }=1.9^{\circ}$

$h=-12 \rightarrow 12$

$k=-12 \rightarrow 12$

$l=-13 \rightarrow 13$

Hydrogen site location: inferred from neighbouring sites

$\mathrm{H}$-atom parameters constrained

$w=1 /\left[\sigma^{2}\left(F_{\mathrm{o}}^{2}\right)+(0.0651 P)^{2}+0.7913 P\right]$

where $P=\left(F_{\mathrm{o}}^{2}+2 F_{\mathrm{c}}^{2}\right) / 3$

$(\Delta / \sigma)_{\max }<0.001$

$\Delta \rho_{\max }=0.61$ e $\AA^{-3}$

$\Delta \rho_{\min }=-0.21{\mathrm{e} \AA^{-3}}^{-3}$ 


\section{Special details}

Geometry. All e.s.d.'s (except the e.s.d. in the dihedral angle between two 1.s. planes) are estimated using the full covariance matrix. The cell e.s.d.'s are taken into account individually in the estimation of e.s.d.'s in distances, angles and torsion angles; correlations between e.s.d.'s in cell parameters are only used when they are defined by crystal symmetry. An approximate (isotropic) treatment of cell e.s.d.'s is used for estimating e.s.d.'s involving 1.s. planes.

Fractional atomic coordinates and isotropic or equivalent isotropic displacement parameters $\left(\AA^{2}\right)$

\begin{tabular}{|c|c|c|c|c|}
\hline & $x$ & $y$ & $z$ & $U_{\text {iso }} * / U_{\text {eq }}$ \\
\hline $\mathrm{C} 1$ & $1.24843(18)$ & $1.05014(17)$ & $0.49190(14)$ & 0.0195 \\
\hline $\mathrm{C} 2$ & $1.05411(17)$ & $0.83773(16)$ & $0.45059(14)$ & 0.0188 \\
\hline $\mathrm{C} 3$ & $1.07636(18)$ & $1.00085(17)$ & $0.32811(14)$ & 0.0194 \\
\hline $\mathrm{C} 4$ & 1.44642 (19) & $1.10652(17)$ & $0.66284(14)$ & $0.0225(4)$ \\
\hline $\mathrm{H} 4$ & 1.4372 & 1.0047 & 0.6426 & $0.027^{*}$ \\
\hline $\mathrm{C} 5$ & $1.61162(19)$ & $1.20826(19)$ & $0.68357(16)$ & $0.0256(4)$ \\
\hline $\mathrm{H} 5 \mathrm{~A}$ & 1.6204 & 1.3094 & 0.6976 & $0.031^{*}$ \\
\hline $\mathrm{H} 5 \mathrm{~B}$ & 1.6569 & 1.1941 & 0.6105 & $0.031^{*}$ \\
\hline C6 & $1.6957(2)$ & $1.1792(2)$ & $0.79337(17)$ & $0.0295(4)$ \\
\hline H6A & 1.6956 & 1.0813 & 0.7755 & $0.035^{*}$ \\
\hline H6B & 1.8018 & 1.2495 & 0.8091 & $0.035^{*}$ \\
\hline $\mathrm{C} 7$ & $1.6240(2)$ & 1.19059 (19) & $0.90564(16)$ & $0.0284(4)$ \\
\hline H7A & 1.6353 & 1.2916 & 0.9295 & $0.034 *$ \\
\hline H7B & 1.6765 & 1.1642 & 0.9737 & $0.034^{*}$ \\
\hline $\mathrm{C} 8$ & $1.4568(2)$ & $1.0913(2)$ & $0.88254(16)$ & $0.0277(4)$ \\
\hline $\mathrm{H} 8 \mathrm{~A}$ & 1.4459 & 0.9896 & 0.8677 & $0.033^{*}$ \\
\hline H8B & 1.4114 & 1.1055 & 0.9556 & $0.033^{*}$ \\
\hline $\mathrm{C} 9$ & $1.3732(2)$ & $1.1221(2)$ & $0.77312(16)$ & $0.0271(4)$ \\
\hline H9A & 1.2663 & 1.0536 & 0.7574 & $0.033^{*}$ \\
\hline H9B & 1.3763 & 1.2212 & 0.7902 & $0.033^{*}$ \\
\hline $\mathrm{C} 10$ & $0.83931(18)$ & $0.61278(17)$ & $0.40889(15)$ & $0.0220(3)$ \\
\hline $\mathrm{H} 10$ & 0.7850 & 0.6696 & 0.3740 & $0.026^{*}$ \\
\hline C11 & $0.7472(2)$ & $0.5209(2)$ & $0.49280(16)$ & $0.0293(4)$ \\
\hline H11A & 0.7268 & 0.5829 & 0.5575 & $0.035^{*}$ \\
\hline H11B & 0.8038 & 0.4697 & 0.5319 & $0.035^{*}$ \\
\hline $\mathrm{C} 12$ & $0.5979(2)$ & $0.4112(2)$ & $0.41799(17)$ & $0.0330(4)$ \\
\hline H12A & 0.5375 & 0.3498 & 0.4718 & $0.040^{*}$ \\
\hline H12B & 0.5395 & 0.4630 & 0.3826 & $0.040^{*}$ \\
\hline $\mathrm{C} 13$ & $0.6271(2)$ & 0.31714 (19) & $0.31667(17)$ & $0.0317(4)$ \\
\hline H13A & 0.5300 & 0.2477 & 0.2689 & $0.038^{*}$ \\
\hline H13B & 0.6814 & 0.2618 & 0.3520 & $0.038^{*}$ \\
\hline $\mathrm{C} 14$ & $0.7213(2)$ & $0.4114(2)$ & $0.23329(16)$ & $0.0297(4)$ \\
\hline H14A & 0.6643 & 0.4622 & 0.1941 & $0.036^{*}$ \\
\hline H14B & 0.7422 & 0.3498 & 0.1685 & $0.036^{*}$ \\
\hline $\mathrm{C} 15$ & 0.87075 (19) & $0.52219(18)$ & $0.30727(15)$ & $0.0243(4)$ \\
\hline H15A & 0.9311 & 0.4715 & 0.3417 & $0.029^{*}$ \\
\hline H15B & 0.9295 & 0.5851 & 0.2535 & $0.029^{*}$ \\
\hline $\mathrm{C} 16$ & 1.09447 (19) & $1.17766(17)$ & $0.19788(15)$ & $0.0219(3)$ \\
\hline H16 & 1.1439 & 1.2490 & 0.2743 & $0.026^{*}$ \\
\hline
\end{tabular}




$\begin{array}{lllll}\text { C17 } & 0.9714(2) & 1.2129(2) & 0.13237(19) & 0.0314(4) \\ \text { H17A } & 0.8992 & 1.2163 & 0.1877 & 0.038^{*} \\ \text { H17B } & 0.9159 & 1.1367 & 0.0612 & 0.038^{*} \\ \text { C18 } & 1.0407(2) & 1.3590(2) & 0.09008(19) & 0.0338(4) \\ \text { H18A } & 0.9602 & 1.3788 & 0.0434 & 0.041^{*} \\ \text { H18B } & 1.0872 & 1.4361 & 0.1620 & 0.041^{*} \\ \text { C19 } & 1.1586(2) & 1.36119(19) & 0.01149(16) & 0.0310(4) \\ \text { H19A } & 1.2046 & 1.4582 & -0.0110 & 0.037^{*} \\ \text { H19B } & 1.1103 & 1.2906 & -0.0643 & 0.037^{*} \\ \text { C20 } & 1.2807(2) & 1.3241(2) & 0.07840(19) & 0.0350(4) \\ \text { H20A } & 1.3352 & 1.3995 & 0.1503 & 0.042^{*} \\ \text { H20B } & 1.3542 & 1.3216 & 0.0241 & 0.042^{*} \\ \text { C21 } & 1.2106(2) & 1.17700(19) & 0.11921(17) & 0.0292(4) \\ \text { H21A } & 1.1629 & 1.1006 & 0.0469 & 0.035^{*} \\ \text { H21B } & 1.2903 & 1.1557 & 0.1653 & 0.035^{*} \\ \text { N1 } & 1.18158(15) & 0.92270(14) & 0.52567(12) & 0.0202(3) \\ \text { N2 } & 0.99553(15) & 0.86893(14) & 0.35006(12) & 0.0203(3) \\ \text { N3 } & 1.20286(15) & 1.09706(14) & 0.39485(12) & 0.0208(3) \\ \text { O1 } & 1.37668(13) & 1.14586(12) & 0.55837(10) & 0.0244(3) \\ \text { O2 } & 0.98237(13) & 0.71122(12) & 0.48406(10) & 0.0227(3) \\ \text { O3 } & 1.01921(13) & 1.03331(12) & 0.22770(10) & 0.0230(3)\end{array}$

Atomic displacement parameters $\left(\AA^{2}\right)$

\begin{tabular}{lllllll}
\hline & $U^{11}$ & $U^{22}$ & $U^{33}$ & $U^{12}$ & $U^{13}$ & $U^{23}$ \\
\hline C1 & $0.0179(7)$ & $0.0191(8)$ & $0.0164(7)$ & $0.0030(6)$ & $0.0012(6)$ & $-0.0001(6)$ \\
C2 & $0.0186(7)$ & $0.0166(7)$ & $0.0189(7)$ & $0.0042(6)$ & $0.0042(6)$ & $0.0024(6)$ \\
C3 & $0.0224(8)$ & $0.0219(8)$ & $0.0154(7)$ & $0.0098(6)$ & $0.0041(6)$ & $0.0042(6)$ \\
C4 & $0.0239(8)$ & $0.0199(8)$ & $0.0180(8)$ & $0.0039(7)$ & $-0.0038(6)$ & $0.0036(6)$ \\
C5 & $0.0223(8)$ & $0.0265(9)$ & $0.0236(8)$ & $0.0046(7)$ & $0.0038(7)$ & $0.0047(7)$ \\
C6 & $0.0204(8)$ & $0.0332(10)$ & $0.0304(9)$ & $0.0062(7)$ & $-0.0004(7)$ & $0.0063(7)$ \\
C7 & $0.0308(9)$ & $0.0273(9)$ & $0.0211(8)$ & $0.0080(7)$ & $-0.0067(7)$ & $0.0020(7)$ \\
C8 & $0.0290(9)$ & $0.0344(10)$ & $0.0206(8)$ & $0.0120(8)$ & $0.0051(7)$ & $0.0091(7)$ \\
C9 & $0.0220(8)$ & $0.0340(9)$ & $0.0238(9)$ & $0.0089(7)$ & $0.0023(7)$ & $0.0075(7)$ \\
C10 & $0.0201(8)$ & $0.0168(8)$ & $0.0218(8)$ & $0.0004(6)$ & $0.0006(6)$ & $0.0019(6)$ \\
C11 & $0.0282(9)$ & $0.0278(9)$ & $0.0198(8)$ & $-0.0018(7)$ & $0.0013(7)$ & $0.0032(7)$ \\
C12 & $0.0284(9)$ & $0.0279(9)$ & $0.0286(9)$ & $-0.0044(7)$ & $0.0026(7)$ & $0.0056(7)$ \\
C13 & $0.0323(10)$ & $0.0191(8)$ & $0.0309(9)$ & $-0.0001(7)$ & $-0.0077(8)$ & $0.0008(7)$ \\
C14 & $0.0334(10)$ & $0.0279(9)$ & $0.0226(8)$ & $0.0104(8)$ & $-0.0032(7)$ & $-0.0032(7)$ \\
C15 & $0.0238(8)$ & $0.0244(8)$ & $0.0208(8)$ & $0.0066(7)$ & $0.0006(6)$ & $0.0014(6)$ \\
C16 & $0.0260(8)$ & $0.0182(8)$ & $0.0186(8)$ & $0.0055(6)$ & $0.0007(6)$ & $0.0054(6)$ \\
C17 & $0.0253(9)$ & $0.0287(9)$ & $0.0407(11)$ & $0.0093(7)$ & $0.0038(8)$ & $0.0138(8)$ \\
C18 & $0.0337(10)$ & $0.0292(10)$ & $0.0416(11)$ & $0.0135(8)$ & $0.0046(8)$ & $0.0152(8)$ \\
C19 & $0.0426(11)$ & $0.0223(8)$ & $0.0223(8)$ & $0.0061(8)$ & $0.0024(8)$ & $0.0076(7)$ \\
C20 & $0.0330(10)$ & $0.0318(10)$ & $0.0415(11)$ & $0.0098(8)$ & $0.0145(8)$ & $0.0143(8)$ \\
C21 & $0.0306(9)$ & $0.0261(9)$ & $0.0344(10)$ & $0.0122(7)$ & $0.0099(8)$ & $0.0110(7)$ \\
N1 & $0.0195(7)$ & $0.0196(7)$ & $0.0167(6)$ & $0.0031(5)$ & $-0.0001(5)$ & $0.0030(5)$ \\
N2 & $0.0191(7)$ & $0.0202(7)$ & $0.0177(6)$ & $0.0046(5)$ & $0.0003(5)$ & $0.0018(5)$ \\
& & & & & &
\end{tabular}




\begin{tabular}{lllllll}
$\mathrm{N} 3$ & $0.0228(7)$ & $0.0183(7)$ & $0.0175(7)$ & $0.0041(6)$ & $0.0019(5)$ & $0.0038(5)$ \\
$\mathrm{O} 1$ & $0.0228(6)$ & $0.0209(6)$ & $0.0204(6)$ & $-0.0001(5)$ & $-0.0041(5)$ & $0.0056(5)$ \\
O2 & $0.0220(6)$ & $0.0188(6)$ & $0.0202(6)$ & $0.0009(5)$ & $-0.0012(4)$ & $0.0041(4)$ \\
O3 & $0.0233(6)$ & $0.0203(6)$ & $0.0202(6)$ & $0.0037(5)$ & $-0.0025(5)$ & $0.0051(4)$ \\
\hline
\end{tabular}

Geometric parameters $\left(\AA,{ }^{o}\right)$

\begin{tabular}{|c|c|c|c|}
\hline $\mathrm{C} 1-\mathrm{N} 1$ & $1.329(2)$ & $\mathrm{C} 11-\mathrm{H} 11 \mathrm{~A}$ & 0.9900 \\
\hline $\mathrm{C} 1-\mathrm{O} 1$ & $1.3338(19)$ & C11-H11B & 0.9900 \\
\hline $\mathrm{C} 1-\mathrm{N} 3$ & $1.334(2)$ & $\mathrm{C} 12-\mathrm{C} 13$ & $1.518(3)$ \\
\hline $\mathrm{C} 2-\mathrm{O} 2$ & $1.3281(19)$ & $\mathrm{C} 12-\mathrm{H} 12 \mathrm{~A}$ & 0.9900 \\
\hline $\mathrm{C} 2-\mathrm{N} 2$ & $1.333(2)$ & $\mathrm{C} 12-\mathrm{H} 12 \mathrm{~B}$ & 0.9900 \\
\hline $\mathrm{C} 2-\mathrm{N} 1$ & $1.340(2)$ & $\mathrm{C} 13-\mathrm{C} 14$ & $1.532(3)$ \\
\hline $\mathrm{C} 3-\mathrm{N} 3$ & $1.326(2)$ & $\mathrm{C} 13-\mathrm{H} 13 \mathrm{~A}$ & 0.9900 \\
\hline $\mathrm{C} 3-\mathrm{O} 3$ & $1.3318(19)$ & $\mathrm{C} 13-\mathrm{H} 13 \mathrm{~B}$ & 0.9900 \\
\hline $\mathrm{C} 3-\mathrm{N} 2$ & $1.339(2)$ & $\mathrm{C} 14-\mathrm{C} 15$ & $1.537(2)$ \\
\hline $\mathrm{C} 4-\mathrm{O} 1$ & $1.4601(19)$ & $\mathrm{C} 14-\mathrm{H} 14 \mathrm{~A}$ & 0.9900 \\
\hline $\mathrm{C} 4-\mathrm{C} 9$ & $1.510(2)$ & C14-H14B & 0.9900 \\
\hline $\mathrm{C} 4-\mathrm{C} 5$ & $1.520(2)$ & $\mathrm{C} 15-\mathrm{H} 15 \mathrm{~A}$ & 0.9900 \\
\hline $\mathrm{C} 4-\mathrm{H} 4$ & 1.0000 & $\mathrm{C} 15-\mathrm{H} 15 \mathrm{~B}$ & 0.9900 \\
\hline $\mathrm{C} 5-\mathrm{C} 6$ & $1.524(2)$ & $\mathrm{C} 16-\mathrm{O} 3$ & $1.4652(19)$ \\
\hline $\mathrm{C} 5-\mathrm{H} 5 \mathrm{~A}$ & 0.9900 & $\mathrm{C} 16-\mathrm{C} 21$ & $1.502(2)$ \\
\hline $\mathrm{C} 5-\mathrm{H} 5 \mathrm{~B}$ & 0.9900 & $\mathrm{C} 16-\mathrm{C} 17$ & $1.514(2)$ \\
\hline $\mathrm{C} 6-\mathrm{C} 7$ & $1.513(3)$ & $\mathrm{C} 16-\mathrm{H} 16$ & 1.0000 \\
\hline $\mathrm{C} 6-\mathrm{H} 6 \mathrm{~A}$ & 0.9900 & $\mathrm{C} 17-\mathrm{C} 18$ & $1.533(2)$ \\
\hline $\mathrm{C} 6-\mathrm{H} 6 \mathrm{~B}$ & 0.9900 & $\mathrm{C} 17-\mathrm{H} 17 \mathrm{~A}$ & 0.9900 \\
\hline $\mathrm{C} 7-\mathrm{C} 8$ & $1.529(2)$ & C17-H17B & 0.9900 \\
\hline $\mathrm{C} 7-\mathrm{H} 7 \mathrm{~A}$ & 0.9900 & $\mathrm{C} 18-\mathrm{C} 19$ & $1.510(3)$ \\
\hline $\mathrm{C} 7-\mathrm{H} 7 \mathrm{~B}$ & 0.9900 & C18-H18A & 0.9900 \\
\hline $\mathrm{C} 8-\mathrm{C} 9$ & $1.526(2)$ & C18-H18B & 0.9900 \\
\hline $\mathrm{C} 8-\mathrm{H} 8 \mathrm{~A}$ & 0.9900 & $\mathrm{C} 19-\mathrm{C} 20$ & $1.524(3)$ \\
\hline $\mathrm{C} 8-\mathrm{H} 8 \mathrm{~B}$ & 0.9900 & C19-H19A & 0.9900 \\
\hline C9-H9A & 0.9900 & С19-H19B & 0.9900 \\
\hline C9-H9B & 0.9900 & $\mathrm{C} 20-\mathrm{C} 21$ & $1.535(2)$ \\
\hline $\mathrm{C} 10-\mathrm{O} 2$ & $1.4680(18)$ & $\mathrm{C} 20-\mathrm{H} 20 \mathrm{~A}$ & 0.9900 \\
\hline $\mathrm{C} 10-\mathrm{C} 15$ & $1.510(2)$ & $\mathrm{C} 20-\mathrm{H} 20 \mathrm{~B}$ & 0.9900 \\
\hline $\mathrm{C} 10-\mathrm{C} 11$ & $1.516(2)$ & $\mathrm{C} 21-\mathrm{H} 21 \mathrm{~A}$ & 0.9900 \\
\hline $\mathrm{C} 10-\mathrm{H} 10$ & 1.0000 & $\mathrm{C} 21-\mathrm{H} 21 \mathrm{~B}$ & 0.9900 \\
\hline $\mathrm{C} 11-\mathrm{C} 12$ & $1.536(2)$ & & \\
\hline $\mathrm{N} 1-\mathrm{C} 1-\mathrm{O} 1$ & $119.36(14)$ & $\mathrm{H} 12 \mathrm{~A}-\mathrm{C} 12-\mathrm{H} 12 \mathrm{~B}$ & 108.1 \\
\hline $\mathrm{N} 1-\mathrm{C} 1-\mathrm{N} 3$ & $127.33(14)$ & $\mathrm{C} 12-\mathrm{C} 13-\mathrm{C} 14$ & $109.90(15)$ \\
\hline $\mathrm{O} 1-\mathrm{C} 1-\mathrm{N} 3$ & $113.31(14)$ & $\mathrm{C} 12-\mathrm{C} 13-\mathrm{H} 13 \mathrm{~A}$ & 109.7 \\
\hline $\mathrm{O} 2-\mathrm{C} 2-\mathrm{N} 2$ & $119.16(14)$ & $\mathrm{C} 14-\mathrm{C} 13-\mathrm{H} 13 \mathrm{~A}$ & 109.7 \\
\hline $\mathrm{O} 2-\mathrm{C} 2-\mathrm{N} 1$ & $114.21(14)$ & $\mathrm{C} 12-\mathrm{C} 13-\mathrm{H} 13 \mathrm{~B}$ & 109.7 \\
\hline $\mathrm{N} 2-\mathrm{C} 2-\mathrm{N} 1$ & $126.63(14)$ & $\mathrm{C} 14-\mathrm{C} 13-\mathrm{H} 13 \mathrm{~B}$ & 109.7 \\
\hline $\mathrm{N} 3-\mathrm{C} 3-\mathrm{O} 3$ & $119.32(14)$ & $\mathrm{H} 13 \mathrm{~A}-\mathrm{C} 13-\mathrm{H} 13 \mathrm{~B}$ & 108.2 \\
\hline $\mathrm{N} 3-\mathrm{C} 3-\mathrm{N} 2$ & $126.75(14)$ & $\mathrm{C} 13-\mathrm{C} 14-\mathrm{C} 15$ & $110.08(14)$ \\
\hline
\end{tabular}




\begin{tabular}{|c|c|c|c|}
\hline $\mathrm{O} 3-\mathrm{C} 3-\mathrm{N} 2$ & $113.93(14)$ & $\mathrm{C} 13-\mathrm{C} 14-\mathrm{H} 14 \mathrm{~A}$ & 109.6 \\
\hline $\mathrm{O} 1-\mathrm{C} 4-\mathrm{C} 9$ & $111.01(14)$ & $\mathrm{C} 15-\mathrm{C} 14-\mathrm{H} 14 \mathrm{~A}$ & 109.6 \\
\hline $\mathrm{O} 1-\mathrm{C} 4-\mathrm{C} 5$ & $105.24(13)$ & $\mathrm{C} 13-\mathrm{C} 14-\mathrm{H} 14 \mathrm{~B}$ & 109.6 \\
\hline $\mathrm{C} 9-\mathrm{C} 4-\mathrm{C} 5$ & $111.64(14)$ & $\mathrm{C} 15-\mathrm{C} 14-\mathrm{H} 14 \mathrm{~B}$ & 109.6 \\
\hline $\mathrm{O} 1-\mathrm{C} 4-\mathrm{H} 4$ & 109.6 & $\mathrm{H} 14 \mathrm{~A}-\mathrm{C} 14-\mathrm{H} 14 \mathrm{~B}$ & 108.2 \\
\hline $\mathrm{C} 9-\mathrm{C} 4-\mathrm{H} 4$ & 109.6 & $\mathrm{C} 10-\mathrm{C} 15-\mathrm{C} 14$ & $109.76(14)$ \\
\hline $\mathrm{C} 5-\mathrm{C} 4-\mathrm{H} 4$ & 109.6 & $\mathrm{C} 10-\mathrm{C} 15-\mathrm{H} 15 \mathrm{~A}$ & 109.7 \\
\hline $\mathrm{C} 4-\mathrm{C} 5-\mathrm{C} 6$ & $109.90(14)$ & $\mathrm{C} 14-\mathrm{C} 15-\mathrm{H} 15 \mathrm{~A}$ & 109.7 \\
\hline $\mathrm{C} 4-\mathrm{C} 5-\mathrm{H} 5 \mathrm{~A}$ & 109.7 & $\mathrm{C} 10-\mathrm{C} 15-\mathrm{H} 15 \mathrm{~B}$ & 109.7 \\
\hline $\mathrm{C} 6-\mathrm{C} 5-\mathrm{H} 5 \mathrm{~A}$ & 109.7 & $\mathrm{C} 14-\mathrm{C} 15-\mathrm{H} 15 \mathrm{~B}$ & 109.7 \\
\hline $\mathrm{C} 4-\mathrm{C} 5-\mathrm{H} 5 \mathrm{~B}$ & 109.7 & $\mathrm{H} 15 \mathrm{~A}-\mathrm{C} 15-\mathrm{H} 15 \mathrm{~B}$ & 108.2 \\
\hline $\mathrm{C} 6-\mathrm{C} 5-\mathrm{H} 5 \mathrm{~B}$ & 109.7 & $\mathrm{O} 3-\mathrm{C} 16-\mathrm{C} 21$ & $109.68(13)$ \\
\hline $\mathrm{H} 5 \mathrm{~A}-\mathrm{C} 5-\mathrm{H} 5 \mathrm{~B}$ & 108.2 & $\mathrm{O} 3-\mathrm{C} 16-\mathrm{C} 17$ & $105.89(13)$ \\
\hline $\mathrm{C} 7-\mathrm{C} 6-\mathrm{C} 5$ & $111.39(15)$ & $\mathrm{C} 21-\mathrm{C} 16-\mathrm{C} 17$ & $111.75(14)$ \\
\hline $\mathrm{C} 7-\mathrm{C} 6-\mathrm{H} 6 \mathrm{~A}$ & 109.3 & $\mathrm{O} 3-\mathrm{C} 16-\mathrm{H} 16$ & 109.8 \\
\hline $\mathrm{C} 5-\mathrm{C} 6-\mathrm{H} 6 \mathrm{~A}$ & 109.3 & $\mathrm{C} 21-\mathrm{C} 16-\mathrm{H} 16$ & 109.8 \\
\hline $\mathrm{C} 7-\mathrm{C} 6-\mathrm{H} 6 \mathrm{~B}$ & 109.3 & $\mathrm{C} 17-\mathrm{C} 16-\mathrm{H} 16$ & 109.8 \\
\hline $\mathrm{C} 5-\mathrm{C} 6-\mathrm{H} 6 \mathrm{~B}$ & 109.3 & $\mathrm{C} 16-\mathrm{C} 17-\mathrm{C} 18$ & $109.82(15)$ \\
\hline $\mathrm{H} 6 \mathrm{~A}-\mathrm{C} 6-\mathrm{H} 6 \mathrm{~B}$ & 108.0 & $\mathrm{C} 16-\mathrm{C} 17-\mathrm{H} 17 \mathrm{~A}$ & 109.7 \\
\hline $\mathrm{C} 6-\mathrm{C} 7-\mathrm{C} 8$ & $111.16(14)$ & $\mathrm{C} 18-\mathrm{C} 17-\mathrm{H} 17 \mathrm{~A}$ & 109.7 \\
\hline $\mathrm{C} 6-\mathrm{C} 7-\mathrm{H} 7 \mathrm{~A}$ & 109.4 & $\mathrm{C} 16-\mathrm{C} 17-\mathrm{H} 17 \mathrm{~B}$ & 109.7 \\
\hline $\mathrm{C} 8-\mathrm{C} 7-\mathrm{H} 7 \mathrm{~A}$ & 109.4 & $\mathrm{C} 18-\mathrm{C} 17-\mathrm{H} 17 \mathrm{~B}$ & 109.7 \\
\hline $\mathrm{C} 6-\mathrm{C} 7-\mathrm{H} 7 \mathrm{~B}$ & 109.4 & $\mathrm{H} 17 \mathrm{~A}-\mathrm{C} 17-\mathrm{H} 17 \mathrm{~B}$ & 108.2 \\
\hline $\mathrm{C} 8-\mathrm{C} 7-\mathrm{H} 7 \mathrm{~B}$ & 109.4 & $\mathrm{C} 19-\mathrm{C} 18-\mathrm{C} 17$ & $111.37(16)$ \\
\hline $\mathrm{H} 7 \mathrm{~A}-\mathrm{C} 7-\mathrm{H} 7 \mathrm{~B}$ & 108.0 & $\mathrm{C} 19-\mathrm{C} 18-\mathrm{H} 18 \mathrm{~A}$ & 109.4 \\
\hline $\mathrm{C} 9-\mathrm{C} 8-\mathrm{C} 7$ & $111.15(14)$ & $\mathrm{C} 17-\mathrm{C} 18-\mathrm{H} 18 \mathrm{~A}$ & 109.4 \\
\hline $\mathrm{C} 9-\mathrm{C} 8-\mathrm{H} 8 \mathrm{~A}$ & 109.4 & $\mathrm{C} 19-\mathrm{C} 18-\mathrm{H} 18 \mathrm{~B}$ & 109.4 \\
\hline $\mathrm{C} 7-\mathrm{C} 8-\mathrm{H} 8 \mathrm{~A}$ & 109.4 & $\mathrm{C} 17-\mathrm{C} 18-\mathrm{H} 18 \mathrm{~B}$ & 109.4 \\
\hline $\mathrm{C} 9-\mathrm{C} 8-\mathrm{H} 8 \mathrm{~B}$ & 109.4 & $\mathrm{H} 18 \mathrm{~A}-\mathrm{C} 18-\mathrm{H} 18 \mathrm{~B}$ & 108.0 \\
\hline $\mathrm{C} 7-\mathrm{C} 8-\mathrm{H} 8 \mathrm{~B}$ & 109.4 & $\mathrm{C} 18-\mathrm{C} 19-\mathrm{C} 20$ & $110.83(15)$ \\
\hline $\mathrm{H} 8 \mathrm{~A}-\mathrm{C} 8-\mathrm{H} 8 \mathrm{~B}$ & 108.0 & $\mathrm{C} 18-\mathrm{C} 19-\mathrm{H} 19 \mathrm{~A}$ & 109.5 \\
\hline $\mathrm{C} 4-\mathrm{C} 9-\mathrm{C} 8$ & $109.41(14)$ & $\mathrm{C} 20-\mathrm{C} 19-\mathrm{H} 19 \mathrm{~A}$ & 109.5 \\
\hline $\mathrm{C} 4-\mathrm{C} 9-\mathrm{H} 9 \mathrm{~A}$ & 109.8 & $\mathrm{C} 18-\mathrm{C} 19-\mathrm{H} 19 \mathrm{~B}$ & 109.5 \\
\hline $\mathrm{C} 8-\mathrm{C} 9-\mathrm{H} 9 \mathrm{~A}$ & 109.8 & $\mathrm{C} 20-\mathrm{C} 19-\mathrm{H} 19 \mathrm{~B}$ & 109.5 \\
\hline $\mathrm{C} 4-\mathrm{C} 9-\mathrm{H} 9 \mathrm{~B}$ & 109.8 & $\mathrm{H} 19 \mathrm{~A}-\mathrm{C} 19-\mathrm{H} 19 \mathrm{~B}$ & 108.1 \\
\hline $\mathrm{C} 8-\mathrm{C} 9-\mathrm{H} 9 \mathrm{~B}$ & 109.8 & $\mathrm{C} 19-\mathrm{C} 20-\mathrm{C} 21$ & $110.28(16)$ \\
\hline $\mathrm{H} 9 \mathrm{~A}-\mathrm{C} 9-\mathrm{H} 9 \mathrm{~B}$ & 108.2 & $\mathrm{C} 19-\mathrm{C} 20-\mathrm{H} 20 \mathrm{~A}$ & 109.6 \\
\hline $\mathrm{O} 2-\mathrm{C} 10-\mathrm{C} 15$ & $109.31(13)$ & $\mathrm{C} 21-\mathrm{C} 20-\mathrm{H} 20 \mathrm{~A}$ & 109.6 \\
\hline $\mathrm{O} 2-\mathrm{C} 10-\mathrm{C} 11$ & $106.48(13)$ & $\mathrm{C} 19-\mathrm{C} 20-\mathrm{H} 20 \mathrm{~B}$ & 109.6 \\
\hline $\mathrm{C} 15-\mathrm{C} 10-\mathrm{C} 11$ & $111.73(14)$ & $\mathrm{C} 21-\mathrm{C} 20-\mathrm{H} 20 \mathrm{~B}$ & 109.6 \\
\hline $\mathrm{O} 2-\mathrm{C} 10-\mathrm{H} 10$ & 109.8 & $\mathrm{H} 20 \mathrm{~A}-\mathrm{C} 20-\mathrm{H} 20 \mathrm{~B}$ & 108.1 \\
\hline $\mathrm{C} 15-\mathrm{C} 10-\mathrm{H} 10$ & 109.8 & $\mathrm{C} 16-\mathrm{C} 21-\mathrm{C} 20$ & $110.14(15)$ \\
\hline $\mathrm{C} 11-\mathrm{C} 10-\mathrm{H} 10$ & 109.8 & $\mathrm{C} 16-\mathrm{C} 21-\mathrm{H} 21 \mathrm{~A}$ & 109.6 \\
\hline $\mathrm{C} 10-\mathrm{C} 11-\mathrm{C} 12$ & $108.91(14)$ & $\mathrm{C} 20-\mathrm{C} 21-\mathrm{H} 21 \mathrm{~A}$ & 109.6 \\
\hline $\mathrm{C} 10-\mathrm{C} 11-\mathrm{H} 11 \mathrm{~A}$ & 109.9 & $\mathrm{C} 16-\mathrm{C} 21-\mathrm{H} 21 \mathrm{~B}$ & 109.6 \\
\hline $\mathrm{C} 12-\mathrm{C} 11-\mathrm{H} 11 \mathrm{~A}$ & 109.9 & $\mathrm{C} 20-\mathrm{C} 21-\mathrm{H} 21 \mathrm{~B}$ & 109.6 \\
\hline $\mathrm{C} 10-\mathrm{C} 11-\mathrm{H} 11 \mathrm{~B}$ & 109.9 & $\mathrm{H} 21 \mathrm{~A}-\mathrm{C} 21-\mathrm{H} 21 \mathrm{~B}$ & 108.1 \\
\hline $\mathrm{C} 12-\mathrm{C} 11-\mathrm{H} 11 \mathrm{~B}$ & 109.9 & $\mathrm{C} 1-\mathrm{N} 1-\mathrm{C} 2$ & $112.84(13)$ \\
\hline
\end{tabular}




\begin{tabular}{|c|c|c|c|}
\hline $\mathrm{H} 11 \mathrm{~A}-\mathrm{C} 11-\mathrm{H} 11 \mathrm{~B}$ & 108.3 & $\mathrm{C} 2-\mathrm{N} 2-\mathrm{C} 3$ & $113.31(14)$ \\
\hline $\mathrm{C} 13-\mathrm{C} 12-\mathrm{C} 11$ & $110.63(16)$ & $\mathrm{C} 3-\mathrm{N} 3-\mathrm{C} 1$ & $113.12(13)$ \\
\hline $\mathrm{C} 13-\mathrm{C} 12-\mathrm{H} 12 \mathrm{~A}$ & 109.5 & $\mathrm{C} 1-\mathrm{O} 1-\mathrm{C} 4$ & $119.74(12)$ \\
\hline $\mathrm{C} 11-\mathrm{C} 12-\mathrm{H} 12 \mathrm{~A}$ & 109.5 & $\mathrm{C} 2-\mathrm{O} 2-\mathrm{C} 10$ & $117.91(12)$ \\
\hline $\mathrm{C} 13-\mathrm{C} 12-\mathrm{H} 12 \mathrm{~B}$ & 109.5 & $\mathrm{C} 3-\mathrm{O} 3-\mathrm{C} 16$ & $118.62(12)$ \\
\hline $\mathrm{C} 11-\mathrm{C} 12-\mathrm{H} 12 \mathrm{~B}$ & 109.5 & & \\
\hline $\mathrm{C} 4-\mathrm{O} 1-\mathrm{C} 1-\mathrm{N} 1$ & $3.6(2)$ & $\mathrm{O} 1-\mathrm{C} 4-\mathrm{C} 5-\mathrm{C} 6$ & $178.67(14)$ \\
\hline $\mathrm{C} 4-\mathrm{O} 1-\mathrm{C} 1-\mathrm{N} 3$ & $-175.80(14)$ & $\mathrm{C} 9-\mathrm{C} 4-\mathrm{C} 5-\mathrm{C} 6$ & $58.2(2)$ \\
\hline $\mathrm{C} 1-\mathrm{O} 1-\mathrm{C} 4-\mathrm{C} 5$ & $157.44(15)$ & $\mathrm{O} 1-\mathrm{C} 4-\mathrm{C} 9-\mathrm{C} 8$ & $-175.68(14)$ \\
\hline $\mathrm{C} 1-\mathrm{O} 1-\mathrm{C} 4-\mathrm{C} 9$ & $-81.64(18)$ & $\mathrm{C} 5-\mathrm{C} 4-\mathrm{C} 9-\mathrm{C} 8$ & $-58.60(19)$ \\
\hline $\mathrm{C} 2-\mathrm{O} 2-\mathrm{C} 10-\mathrm{C} 15$ & $87.08(17)$ & $\mathrm{C} 4-\mathrm{C} 5-\mathrm{C} 6-\mathrm{C} 7$ & $-55.8(2)$ \\
\hline $\mathrm{C} 10-\mathrm{O} 2-\mathrm{C} 2-\mathrm{N} 1$ & $178.32(14)$ & $\mathrm{C} 5-\mathrm{C} 6-\mathrm{C} 7-\mathrm{C} 8$ & $55.0(2)$ \\
\hline $\mathrm{C} 10-\mathrm{O} 2-\mathrm{C} 2-\mathrm{N} 2$ & $-1.2(2)$ & $\mathrm{C} 6-\mathrm{C} 7-\mathrm{C} 8-\mathrm{C} 9$ & $-55.5(2)$ \\
\hline $\mathrm{C} 2-\mathrm{O} 2-\mathrm{C} 10-\mathrm{C} 11$ & $-152.09(15)$ & $\mathrm{C} 7-\mathrm{C} 8-\mathrm{C} 9-\mathrm{C} 4$ & $56.8(2)$ \\
\hline $\mathrm{C} 3-\mathrm{O} 3-\mathrm{C} 16-\mathrm{C} 17$ & $-148.75(15)$ & $\mathrm{O} 2-\mathrm{C} 10-\mathrm{C} 11-\mathrm{C} 12$ & $-177.83(14)$ \\
\hline $\mathrm{C} 16-\mathrm{O} 3-\mathrm{C} 3-\mathrm{N} 2$ & $177.09(15)$ & $\mathrm{C} 11-\mathrm{C} 10-\mathrm{C} 15-\mathrm{C} 14$ & $58.48(19)$ \\
\hline $\mathrm{C} 16-\mathrm{O} 3-\mathrm{C} 3-\mathrm{N} 3$ & $-3.1(2)$ & $\mathrm{C} 15-\mathrm{C} 10-\mathrm{C} 11-\mathrm{C} 12$ & $-58.6(2)$ \\
\hline $\mathrm{C} 3-\mathrm{O} 3-\mathrm{C} 16-\mathrm{C} 21$ & $90.51(18)$ & $\mathrm{O} 2-\mathrm{C} 10-\mathrm{C} 15-\mathrm{C} 14$ & $176.06(13)$ \\
\hline $\mathrm{C} 2-\mathrm{N} 1-\mathrm{C} 1-\mathrm{O} 1$ & $-179.98(15)$ & $\mathrm{C} 10-\mathrm{C} 11-\mathrm{C} 12-\mathrm{C} 13$ & $58.4(2)$ \\
\hline $\mathrm{C} 1-\mathrm{N} 1-\mathrm{C} 2-\mathrm{O} 2$ & $-178.09(15)$ & $\mathrm{C} 11-\mathrm{C} 12-\mathrm{C} 13-\mathrm{C} 14$ & $-58.9(2)$ \\
\hline $\mathrm{C} 2-\mathrm{N} 1-\mathrm{C} 1-\mathrm{N} 3$ & $-0.7(3)$ & $\mathrm{C} 12-\mathrm{C} 13-\mathrm{C} 14-\mathrm{C} 15$ & $58.0(2)$ \\
\hline $\mathrm{C} 1-\mathrm{N} 1-\mathrm{C} 2-\mathrm{N} 2$ & $1.4(3)$ & $\mathrm{C} 13-\mathrm{C} 14-\mathrm{C} 15-\mathrm{C} 10$ & $-57.36(19)$ \\
\hline $\mathrm{C} 2-\mathrm{N} 2-\mathrm{C} 3-\mathrm{N} 3$ & $0.1(3)$ & $\mathrm{O} 3-\mathrm{C} 16-\mathrm{C} 17-\mathrm{C} 18$ & $-176.46(14)$ \\
\hline $\mathrm{C} 3-\mathrm{N} 2-\mathrm{C} 2-\mathrm{O} 2$ & $178.35(15)$ & $\mathrm{C} 21-\mathrm{C} 16-\mathrm{C} 17-\mathrm{C} 18$ & $-57.1(2)$ \\
\hline $\mathrm{C} 3-\mathrm{N} 2-\mathrm{C} 2-\mathrm{N} 1$ & $-1.1(3)$ & $\mathrm{O} 3-\mathrm{C} 16-\mathrm{C} 21-\mathrm{C} 20$ & $175.18(14)$ \\
\hline $\mathrm{C} 2-\mathrm{N} 2-\mathrm{C} 3-\mathrm{O} 3$ & $179.86(14)$ & $\mathrm{C} 17-\mathrm{C} 16-\mathrm{C} 21-\mathrm{C} 20$ & $58.1(2)$ \\
\hline $\mathrm{C} 1-\mathrm{N} 3-\mathrm{C} 3-\mathrm{O} 3$ & $-179.28(15)$ & $\mathrm{C} 16-\mathrm{C} 17-\mathrm{C} 18-\mathrm{C} 19$ & $55.9(2)$ \\
\hline $\mathrm{C} 3-\mathrm{N} 3-\mathrm{C} 1-\mathrm{N} 1$ & $-0.2(3)$ & $\mathrm{C} 17-\mathrm{C} 18-\mathrm{C} 19-\mathrm{C} 20$ & $-56.4(2)$ \\
\hline $\mathrm{C} 3-\mathrm{N} 3-\mathrm{C} 1-\mathrm{O} 1$ & $179.16(15)$ & $\mathrm{C} 18-\mathrm{C} 19-\mathrm{C} 20-\mathrm{C} 21$ & $56.6(2)$ \\
\hline $\mathrm{C} 1-\mathrm{N} 3-\mathrm{C} 3-\mathrm{N} 2$ & $0.5(3)$ & $\mathrm{C} 19-\mathrm{C} 20-\mathrm{C} 21-\mathrm{C} 16$ & $-57.1(2)$ \\
\hline
\end{tabular}

Hydrogen-bond geometry $\left(\AA,{ }^{\circ}\right)$

\begin{tabular}{lllll}
\hline$D-\mathrm{H} \cdots A$ & $D-\mathrm{H}$ & $\mathrm{H} \cdots A$ & $D \cdots A$ & $D-\mathrm{H} \cdots A$ \\
\hline $\mathrm{C} 12-\mathrm{H} 12 A \cdots \mathrm{O} 1^{\mathrm{i}}$ & 0.99 & 2.45 & $3.413(2)$ & 164 \\
$\mathrm{C} 9-\mathrm{H} 9 A \cdots \mathrm{O} 3^{\text {ii }}$ & 0.99 & 2.60 & $3.528(2)$ & 156 \\
$\mathrm{C} 10-\mathrm{H} 10 \cdots \mathrm{O} 1^{\mathrm{ii}}$ & 1.00 & 2.95 & $3.787(2)$ & 142 \\
$\mathrm{C} 5-\mathrm{H} 5 B \cdots \mathrm{N} 1^{\mathrm{iii}}$ & 0.99 & 2.77 & $3.684(2)$ & 154 \\
\hline
\end{tabular}

Symmetry codes: (i) $x-1, y-1, z$; (ii) $-x+2,-y+2,-z+1$; (iii) $-x+3,-y+2,-z+1$. 